\title{
A New Injectivity Prediction Model for Early Polymer Injection
}

\author{
Xiang'an Lu, Hanqiao Jiang, and Yanli Pei
}

\begin{abstract}
Early polymer injection has become an effective way to improve oil recovery in deep offshore fields. Since the reservoir will still be high in oil saturation during this process, a detailed description of water-oil two-phase flow is required to accurately predict polymer injectivity of the reservoir. A polymer fractional flow model was first derived by including polymer rheology effects and two phase flow resistance factor. Water saturation profile was then proposed as four self-sharpening fronts to describe the detailed fluid saturation distribution. The polymer injectivity prediction model for both monolayer and multilayer reservoirs was finally established by separating the pressure drop into several parts based on the saturation profile. Results showed the new model improved the accuracy and efficiency of injectivity prediction in early polymer injection. This model is more like a miniature numerical simulator for early polymer injection that can output the dynamic polymer injectivity with the input of only a few pieces of field data that can be acquired easily.
\end{abstract}

Index Terms-Polymer injection, fractional flow theory, injectivity model, multilayer reservoir.

\section{INTRODUCTION}

Polymer solutions are widely used in chemical EOR processes such as polymer flood and surfactant/polymer flood. Polymer injectivity is a critical factor to evaluate polymer-flooding applicability in field conditions.

The complex physics of polymer rheology in porous media has been the subject of intensive research [1], [2]. Seright [3] presented a series of studies on polymer adsorption, polymer rheology and polymer mechanical degradation in which xanthan and HPAM were the two specific solutions of their study. During most polymer floods process, injecting polymer solutions at a designed injection rate based on the above observations may cause above formation-parting pressure and may induce fractures or fracture-like features [4]-[8].

To obtain a better estimate of the polymer injectivity, many researchers acquiesced to the common practice of dividing the total pressure drop in the reservoir into several parts.

1) The "entrance pressure drop" associated with polymer mechanical degradation at the sandface,

2) The pressure drop associated with dilatants or viscoelastic polymer behavior near a wellbore,

3) The pressure drop associated with polymer solutions flowing at low fluxes and exhibiting a Newtonian or

Manuscript received February 15, 2015; revised April 30, 2015.

The authors are with the Key Laboratory of Petroleum Engineering of the Ministry of Education, China University of Petroleum, Beijing, China (e-mail: 345369400@qq.com). flux-independent behavior,

4) Pressure drop associated with the flow of brine and oil.

Shuler [9] conducted a series of studies on improving polymer injectivity. Results showed that for a single-layer radial flow model where each polymer and brine slug was assumed to move in a piston-like displacement, at any instant, the total pressure drop may be expressed as the sum of:

1) The pressure drop of oil/brine zone ahead of the polymer zone,

2) The pressure drop across the polymer zone,

3) The pressure drop caused by skin effects.

These models assume that the flooding process is piston-like, while none of them consider the actual oil saturation distribution in reservoir. Hall's method has a smoothing effect on the data, data acquisition for Hall plot is inexpensive, but it could not calculate the injectivity of polymer solutions under variable injection rate and therefore is not suitable for situations where short pre-water flooding happens.

This paper develops a polymer injectivity prediction model that accounts for oil saturation distribution, multilayer injection capacity and fluid dynamic characterization. The model improves the accuracy and efficiency in polymer injectivity prediction, and especially for deep offshore field that have been exposed to early polymer injection. To some extent, this model is more like a simplistic numerical simulator which can output dynamic polymer injectivity information with just a few extra field data input. Thus, it's efficient and cost saving during field tests.

\section{POLYMER INJECTIVITY MODEL}

\section{A. Fractional Flow in Polymer Floods}

Characteristic velocity: Based on the classic Buckley-Leverett theory [10], the continuity equation for water phase of one dimensional linear system can be written as:

$$
\frac{\partial S_{w}}{\partial t}+\frac{q}{A \phi} \frac{d f_{w}}{d S_{w}} \frac{\partial S_{w}}{\partial x}=0
$$

The characteristic velocity of a displacement front with constant saturation is

$$
\left(\frac{\partial x}{\partial t}\right)_{S_{w}}=\frac{q}{A \phi} \frac{d f_{w}}{d S_{w}}
$$

The characteristic velocity of water and polymer are equal [11] at the front of polymer flooding: 


$$
\frac{f_{w}^{p}\left(S_{w 3}\right)-f_{w}\left(S_{w 2}\right)}{S_{w 3}-S_{w 2}}=\left.\frac{\partial f_{w}^{p}}{\partial S_{w}}\right|_{S_{w}=S_{w 2}}=\frac{f_{w}^{p}\left(S_{w 2}\right)}{S_{w 2}+D_{p}}
$$

where $D_{p}$ is the polymer retardation factor.

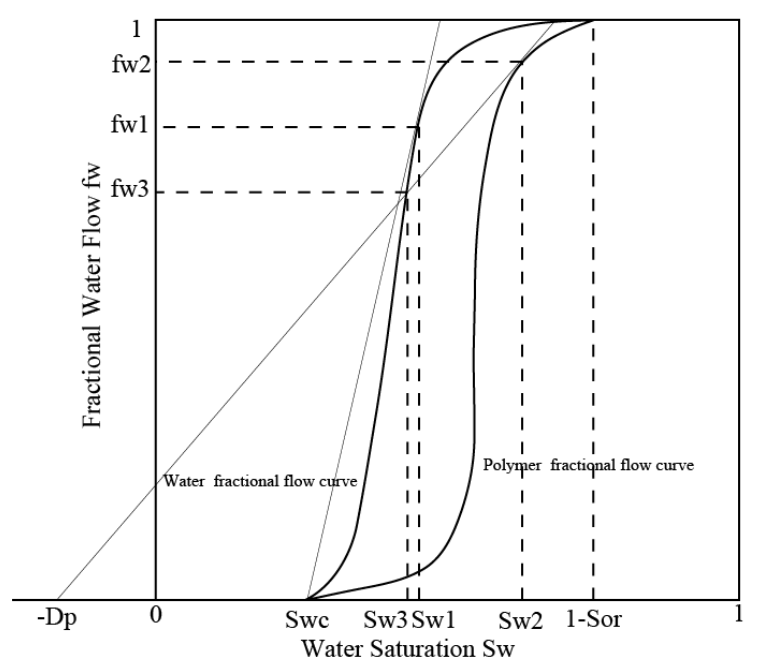

Fig. 1. Fractional flow curves for the water and polymer floods.

Based on (3), water saturation $S_{w 3}$ at the polymer shock front can be obtained graphically by drawing a line segment from the point $\left(-D_{p}, 0\right)$ tangent to the polymer fractional flow curve. This straight line intersects the water fractional flow curve at point $\left(S_{w 3}, f_{w}\left(S_{w 3}\right)\right)$. Similarly, water saturation at the flood frontal $S_{w 1}$ can be obtained by drawing a straight line from the point $\left(S_{w c}, 0\right)$ tangent to the water fractional flow curve Fig. 1.

Saturation Profile: The saturation profile of the simplified polymer flooding can be illustrated in Fig. 2. There are four self-sharpening fronts in the water phase saturation profile compared with the single shock front of Buckley-Leverett theory.

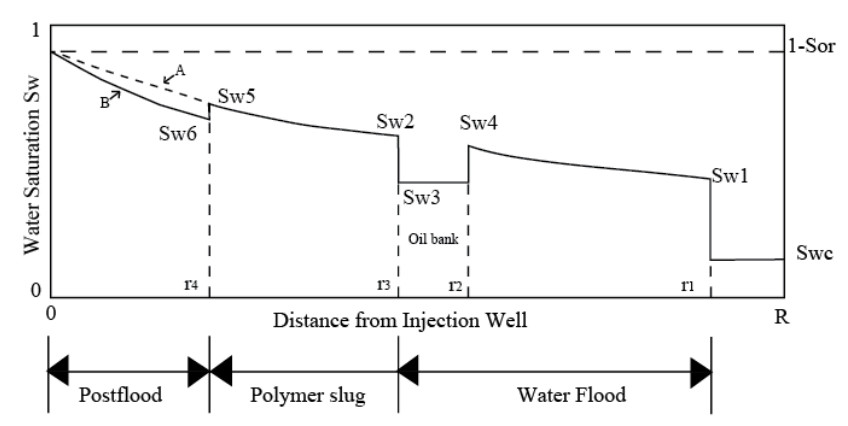

Fig. 2. Water saturation profile of early polymer flooding.

\section{B. Injectivity Model}

Injectivity index: Injectivity index is defined as the injection rate divided by the injection pressure drop

$$
I=Q / \Delta P
$$

According to literature, the injection pressure drop can be divided into several parts based on division of regions in Fig. 2. For 1-D radial flow, the injection pressure drop is given by

$$
\Delta P=\Delta P_{r_{w} 4}+\Delta P_{43}+\Delta P_{32}+\Delta P_{21}+\Delta P_{1 R}
$$

The pressure drop in any region has the same form

$$
\Delta P_{i j}=\frac{Q}{2 \pi h} \int_{r_{i}}^{r_{j}}\left[\frac{1}{r K\left(\frac{k_{r o}\left(S_{w}\right)}{\mu_{o}}+\frac{k_{r w}\left(S_{w}\right)}{\mu_{w}}\right)}\right] d r
$$

Injectivity prediction for multilayer reservoirs: Fig. 3 illustrates the multilayer reservoir schematic model of this study. The reservoir consists of two or more independent layers, each having the same outer radius but different geological properties such as permeability, thickness, porosity and skin. Each layer is assumed to be homogeneous and isotropic, and is filled with slightly compressible fluid. The reservoir is initially at a uniform pressure and the total injection rate is measured at initial reservoir conditions. Gravity and capillary pressure effects are negligibly insignificant.

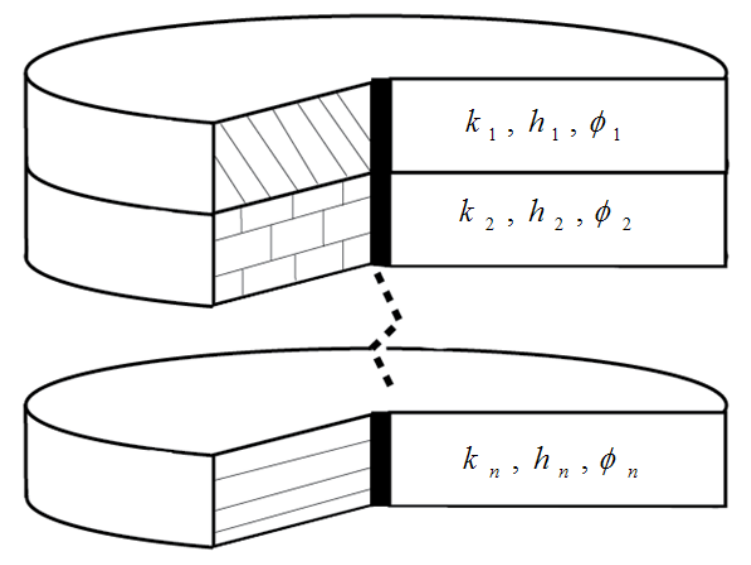

Fig. 3. Idealized multilayer reservoir model.

Here, we define flow rate $q_{i}$ for a single reservoir layer. The total flow rate is the summation of flow rate of all reservoir layers.

$$
Q=\sum_{i=1}^{m} q_{i}
$$

For convenience, we define flow resistance $R_{i}$, the inverse of injectivity, for a single reservoir layer.

$$
\Delta P_{i}=q_{i} R_{i}=q_{i} / I_{i}
$$

Assume the reservoir is initially at a uniform pressure and we have

$$
q_{1}: q_{2} \ldots: q_{m}=\frac{1}{R_{1}}: \frac{1}{R_{2}} \ldots: \frac{1}{R_{m}}
$$

Combining (19), (20) and (21), the injection rate for every layer is

$$
q_{i}=\frac{1 / R_{i}}{\sum_{k=1}^{m} 1 / R_{k}} Q
$$

Model Solving Process: Time and spatial discretization is a must to solve this mathematical model. 


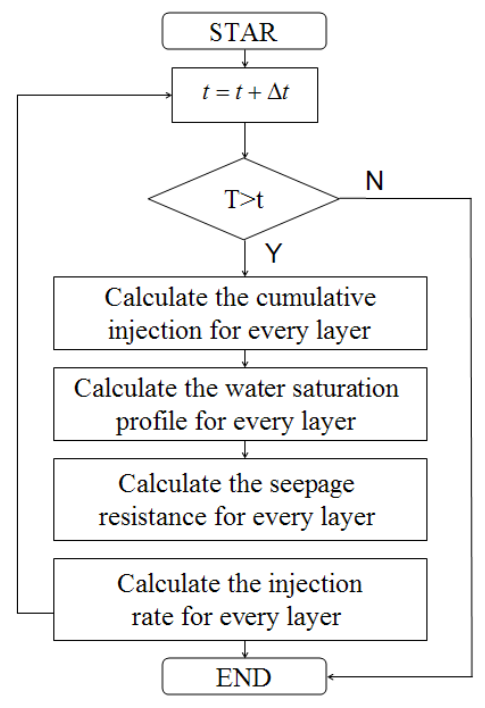

Fig. 4. Calculation flow chart.

As shown in Fig. 4, during each iteration of each time step, four steps needs to be taken care of in succession for each layer of the reservoir:

1) Calculate their current cumulative injection volume,

2) Calculate their water saturation profile,

3) Calculate their flow resistance based on step 2,

4) Calculate the injection rate of every layer according to the ratio of flow resistance of neighboring layers.

To guarantee accuracy, temporal and spatial steps should be small enough to make sure that both pressure field and saturation field are numerically stable within a specific time step.

\section{MOdel VALidATION}

We apply the injectivity prediction model developed above to estimate the injectivity of AP-P4 solution during linear core flooding. AP-P4 is a high molar mass hydrophobic association polymer. Prediction results will be compared with experimental results of steady-state polymer flooding involving sand pack. The injection pressure drop for linear system is given by

$$
\Delta P=\Delta P_{04}+\Delta P_{43}+\Delta P_{32}+\Delta P_{21}+\Delta P_{1 L}
$$

An unconsolidated sand pack was used to validate the injectivity prediction model. The injection rate was first set to be $0.1 \mathrm{~mL} / \mathrm{min}$. When the water cut at the outlet reaches 0.8 , the polymer solutions AP-P4 with $1750 \mathrm{ppm}$ in a salinity of $5855 \mathrm{mg} / \mathrm{L}$ were forced into the sand pack for $0.3 \mathrm{PV}$. Polymer viscosity was $10 \mathrm{mPa} \cdot \mathrm{s}$ at zero shear rate after shearing pretreatment.

Fig. 5 (a) shows the observed and calculated pressure drop during the polymer flooding. The calculated results match the observed results reasonably well except for the injection volume of $0 \sim 0.3 \mathrm{PV}$ due to unsteady-state pressure in the sand pack. Fig. 5 (b) shows the change of water cut at the outlet. The largest discrepancy lies in decrease part of the water cut. There are two factors contributing to this discrepancy. First, all fronts have been calculated as a sudden shock in the model. Second, the saturation of oil bank is considered as a constant.

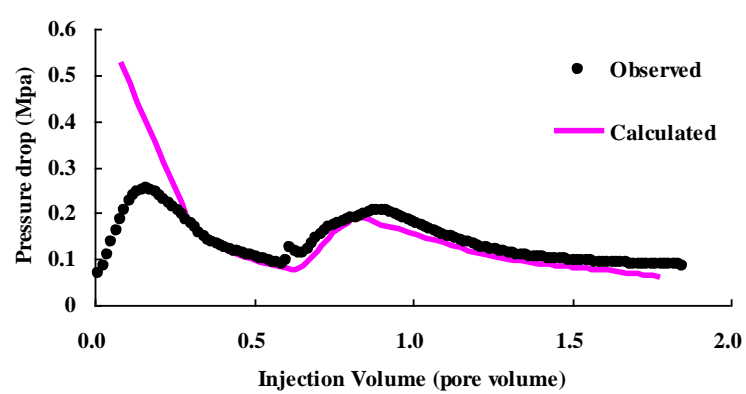

(a)

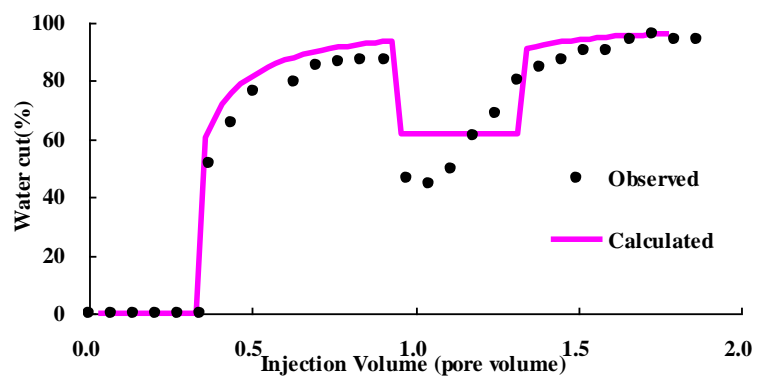

(b)

(a) Pressure drop and (b) water cut at the outlet for sand pack flooding Idealized multilayer reservoir model.

Fig. $5.2079 \mathrm{mD}$ sand pack, porosity $=0.29$, length $=50 \mathrm{~cm}$, diameter $=2.5 \mathrm{~cm}$, water viscosity $=0.5 \mathrm{mPa} \cdot \mathrm{s}$, oil viscosity $=70 \mathrm{mPa} \cdot \mathrm{s}, \mathrm{Fr}=22, \mathrm{Frr}=1.1$.

\section{MODEL APPLICATION}

The single-well polymer-injection pilot was implemented there with a basic well pattern of standard five-point well pattern which includes four production wells and one central injection well. The average distance between oil well and water well is 250 meters. A1 well, a former production well, was later transformed into an injection well according to the pilot plan. At the beginning of polymer injection, the composite water cut of the pilot test area was about $50 \%$.

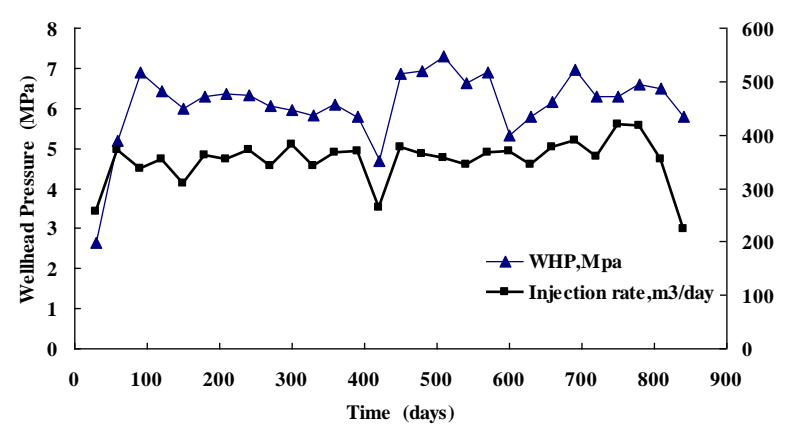

Fig. 6. Wellhead data of A1 well.

Fig. 6 shows the change of injection rate and wellhead pressure of A1 well. After transformed to injection well, A1 well injected water for the first month at the rate of 250 $\mathrm{m}^{3} /$ day and then injected polymer for the second month at the rate of $350 \mathrm{~m}^{3} /$ day along with the wellhead pressure rising from 2.7 MPa to 6.9 MPa. Then the wellhead pressure maintained at $6 \mathrm{MPa}$ with the $4 \mathrm{MPa}$ pressure rise caused by 
polymer injection.

\begin{tabular}{llll}
\multicolumn{3}{l}{ TABLE I: PHYSICAL PROPERTIES OF EVERY LAYER } \\
\hline \hline Layer & Thickness / m & Porosity & Permeability /mD \\
\hline 1 & 6.4 & 0.31 & 1652.9 \\
2 & 4.7 & 0.32 & 1423.2 \\
3 & 7.5 & 0.31 & 5523.3 \\
4 & 4.4 & 0.33 & 4508.8 \\
5 & 2.0 & 0.31 & 501.0 \\
\hline \hline
\end{tabular}

The physical properties of all water-absorption layers are listed in Table I, the total thickness of all water-absorption layers is 25 meters, average permeability is $3187 \mathrm{mD}$, water phase viscosity is $0.5 \mathrm{mPa} \cdot \mathrm{s}$, and oil phase viscosity is 60 $\mathrm{mPa} \cdot \mathrm{s}$.

The effect of polymer injection occasion: From the water flooding simulation of layer 5, the change of outlet water cut is displayed in Fig. 7 (a). The water cut increased sharply when total injection volume reached $0.075 \mathrm{PV}$, indicating water breakthrough. Fig. 7 (b) reveals the pressure change of water flooding with varying injection volume. With the increase of outlet water saturation, average water saturation of the whole model increased, flow resistance of the two-phase flow decreased, and flow pressure difference of the reservoir reduced.

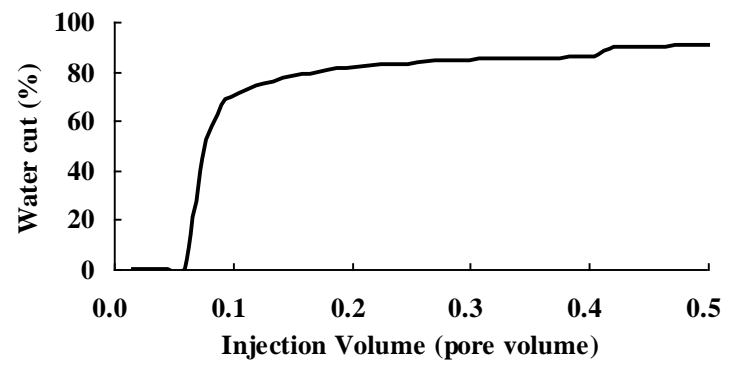

(a)

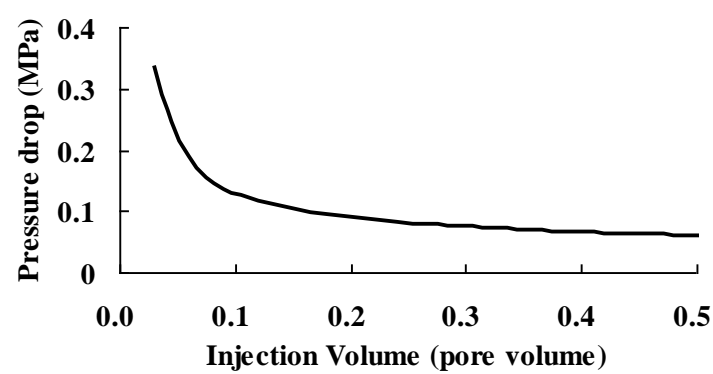

(b)

Fig. 7. (a) Outlet water cut vs. PV injected. (b) Pressure drop vs. PV injected.

The effect of water saturation on the polymer injectivity was considered in this study. The polymer injectivity changes were simulated separately with respect to different polymer slug size. Fig. 8 (a) showed that the pressure drop rose up to almost the same value of 1.6 MPa for different polymer injection occasion. But for the polymer injectivity, the later polymer flooding happened, the greater the injectivity decreased Fig. 8 (b). For well group A1, the average water cut was around $50 \%$ and the total injected PV of water flooding was 0.088 in the following analysis.

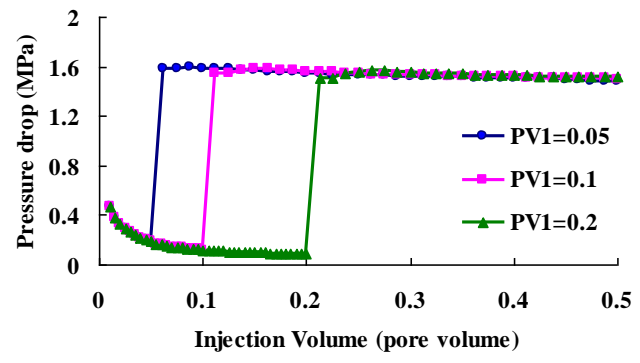

(a)

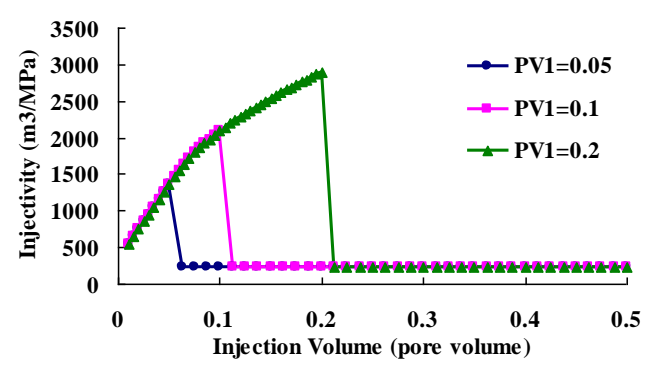

(b)

Fig. 8. (a) Pressure drop vs. PV injected. (b) Injectivity index vs. PV injected.

Wellhead Pressure: Wellhead pressure (WHP) $P_{w}$ is

$$
P_{w}=\Delta P+P_{e}-\Delta P_{H}
$$

where $P_{e}$ is the reservoir pressure; $\Delta P_{H}$ is the hydrostatic column pressure difference in the wellbore caused by gravity, $\Delta P$ is the injection pressure difference which is exactly the pressure difference between flowing bottomhole pressure and reservoir pressure. The dynamic change of WHP is influenced by average reservoir pressure, injection pressure difference and hydrostatic column pressure difference. The hydrostatic column pressure difference in the wellbore can be assumed to be constant if we ignore the polymer influence on the density of water phase. Average reservoir pressure is greatly affected by injection-production ratio. The injection-production ratio of well pattern $\mathrm{A} 1$ is equal to one so that the average reservoir pressure is remained unchanged.

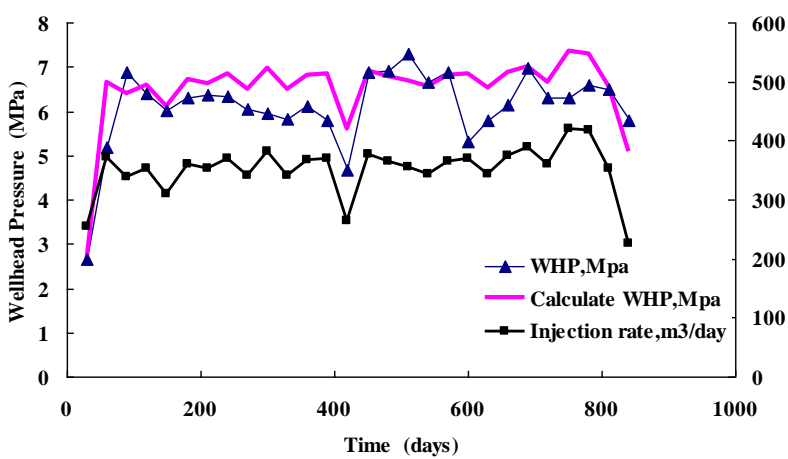

Fig. 9. Match of well head pressure using polymer injection model $(P V 1=0.088, R F=80, S=1.3)$.

Compared with water injection, the biggest difference of polymer injection lies in the injection pressure difference. We arrive at the conclusion that the change in WHP is almost equal to that of the injection pressure difference. As shown in Fig. 9, we calculated the WHP when the injection pressure is increased by 2.5 overall and compare the pressure change 
before and after polymer injection. During the WHP match process, water injection PV number is equal to 0.088, resistance factor is 80 and skin factor is 1.3 . Results show that the calculated WHP change correlates well with the solution injection volume. The WHP and the calculated WHP share the same reservoir pressure rise trend around $4 \mathrm{MPa}$ after the polymer solution injection. Limited by the assumptions above, it is acceptable that the calculated WHP cannot completely fit with the field WHP.

Injection profile: Since the wellhead pressure can be obtained much more easily compared with bottom hole pressure drop, apparent injectivity index showed in (13) is more convenient and practical for analyzing the single well injectivity.

$$
I_{a}=Q /\left(h P_{w}\right)
$$

As shown in Fig. 10, apparent injectivity index for both water flooding and polymer flooding were calculated by injectivity prediction model. Results showed that the apparent injectivity index of well A1 has declined to 2.2 $\mathrm{m}^{2} /$ day/MPa from $3.8 \mathrm{~m}^{2} /$ day/MPa through polymer injection. For single layers, their changes are rather significant, especially layer 3 which has the highest permeability. Thus, polymer injection has a certain even effect on the injection profile.

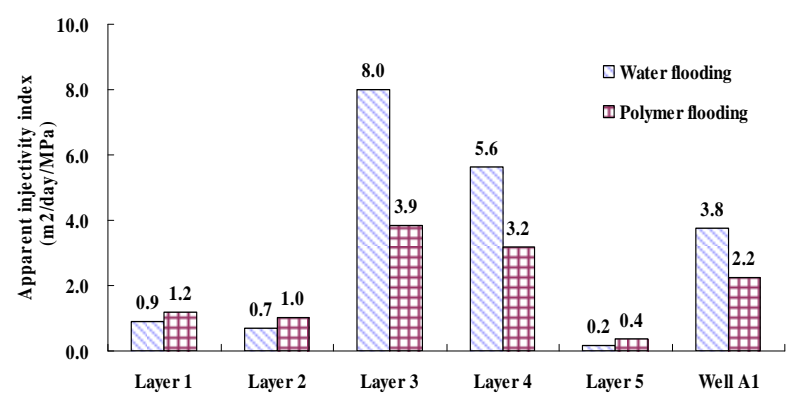

Fig. 10. Comparison of apparent injectivity index $(\mathrm{PV} 1=0.088, \mathrm{RF}=80$, $S=1.3)$.

\section{SUMMARY AND CONCLUSIONS}

Early polymer injection is quite different from conventional polymer injection. Both polymer rheology effects and two phase flow resistance coefficient was included in the newly developed injectivity prediction model.

A semi-analytical model was developed to predict polymer-injectivity change of a single well. The proposed model was verified by one-dimensional core experiments as well as a multilayer reservoir experiment.

The polymer injectivity prediction model was applied to a two-parallel-tube oil displacement core example. Calculation results showed close match with experimental data of the one-dimensional sand pack polymer flooding.

Finally, the model accepts readily available parameters, e.g. well head pressure, injection rate, cumulative injection, reservoir properties, et al, and outputs dynamic polymer injectivity. Therefore, the polymer injectivity prediction model is akin to a miniature numerical simulator.

\section{NOMENCLATURE}

A cross-sectional area

$f_{w}$ fractional flow curve

$f_{w}^{p}$ polymer fractional flow curve

$h$ thickness

$I$ injectivity index

$I_{a} \quad$ apparent injectivity index

$K$ permeability

$k_{r \alpha}$ relative permeability of phase $\alpha$

$P_{e} \quad$ average formation pressure

$P_{w} \quad$ wellhead pressure

$\Delta P$ pressure drop

$\Delta P_{i j}$ pressure drop between location $\mathrm{i}$ and $\mathrm{j}$

$\Delta P_{H}$ hydrostatic column pressure difference

$\Delta P_{s}$ additional pressure drop

$P V_{1}$ the size of blank water flood

$P V_{2}$ the size of polymer slug

$P V_{3}$ the size of post water flood

$q$ injection rate of layer $i$

$Q \quad$ injection rate of well

$r$ radial distance

$r_{w} \quad$ well radius

$R \quad$ radius of idealized reservoir model

$R_{i} \quad$ percolation resistance of layer $i$

$S$ skin factor

$S_{\alpha}$ saturation of the phase $\alpha$

$t$ time

$T$ total time

$\Delta t$ time step

$\phi$ porosity for polymer solution

Subscripts:

$\alpha$ phase ( $\alpha=w$ for water, $\alpha=o$ for oil)

$i, j$ location number or layer number

\section{REFERENCES}

[1] P. L. Bondor, G. J. Hirasaki, and M. J. Tham, "Mathematical simulation of polymer flooding in complex reservoirs," SPE J, vol. 12, no. 5, pp. 369-382, 1972.

[2] R. S. Seright, "The effects of mechanical degradation and viscoelastic behavior on injectivity of polyacrylamide solutions," SPEJ, pp. 475-485, June 1983.

[3] R. S. Seright, M. Seheult, and T. Talashek, "Injectivity characteristics for EOR polymers,” SPE J., vol. 12, no. 5, pp. 783-792, 2009.

[4] M. Khodaverdian, T. Sorop, S. Postif et al., "Polymer flooding in unconsolidated sand formations: fracturing and geomechanical considerations," paper SPE 121840 presented at the EUROPEC/EAGE Annual Conference and Exhibition, Amsterdam, the Netherlands, June, 2009.

[5] P. J. van den Hoek, H. Mahani, T. G. Sorop et al., "Application of injection fall-off analysis in polymer flooding,” paper PE 154376 presented at the SPE EUROPEC/EAGE Annual Conference, Copenhagen, Denmark, 4-7 June, 2012.

[6] T. W. Teklu, W. Alameri, R. M. Graves, et al., "Geomechanics considerations in enhanced oil recovery,” paper SPE 162701 presented at the SPE Canadian Unconventional Resources Conference, Calgary, Alberta, Canada, 30 October-1 November, 2012.

[7] T. Clemens, M. Deckers, M. Kornberger et al., "Basic rheological behavior of zanthan polysaccharide solutions in porous media,” Austria 
paper SPE 164904 presented at the 75th EAGE Conference \& Exhibition Incorporating SPE EUROPEC, London, UK, 10-13 June, 2013.

[8] R. N. Manichand, K. P. M. S. Let, L. Gil, et al., "Effective propagation of HPAM solutions through the tambaredjo reservoir during a polymer flood," paper SPE 164121 presented at the 2013 SPE International Symposium on Oilfield Chemistry, The Woodlands, Texas, 8-10 April, 2013.

[9] P. J. Shuler, D. L. Kuehen et al., "Improving polymer injectivity at west coyote field, California," SPE Reservoir Engineering, August, 1987.

[10] S. E. Buckley and M. C. Leverett, "Mechanism of Fluid Displacement in Sands," AIME, vol. 146, pp. 107-116, 1942.

[11] G. A. Pope, "The Application of Fractional Flow Theory to Enhanced Oil Recovery,” Society of Petroleum Engineers Journal, pp. 191-205, June 1980.

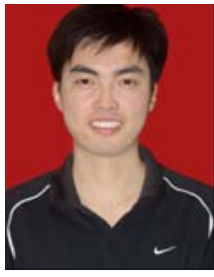

Xiang'an Lu is a PhD student in China University of Petroleum, Beijing, China, who is expected to receive his $\mathrm{PhD}$ degree of oil and gas field development engineering in July 2015. His main research area is reservoir engineering, reservoir numerical simulation, EOR. He is an author or co-author of over 10 papers. $\mathrm{Dr}$. Lu is also a student member of society of petroleum engineers.

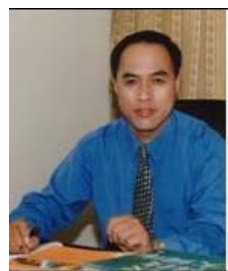

Hanqiao Jiang is a professor in China University of Petroleum, Beijing, China. His main research area is reservoir engineering, water shutoff, profile control, enhanced oil recovery and reservoir numerical simulation. He is an author or co-author of over 200 papers. Now he is a chief of national basic research program of China "973" funded by ministry of science and technology run by five universities and research

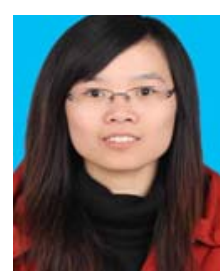

Yanli Pei is a graduate student in China University of Petroleum, Beijing. Her main research area is reservoir engineering and polymer flooding. She is also a student member of society of petroleum engineers. 